\title{
КЛИНИЧЕСКИЕ ХАРАКТЕРИСТИКИ И ИСХОДЫ У ПАЦИЕНТОВ С НОВОЙ КОРОНАВИРУСНОЙ ИНФЕКЦИЕЙ И ИНФАРКТОМ МИОКАРДА
}

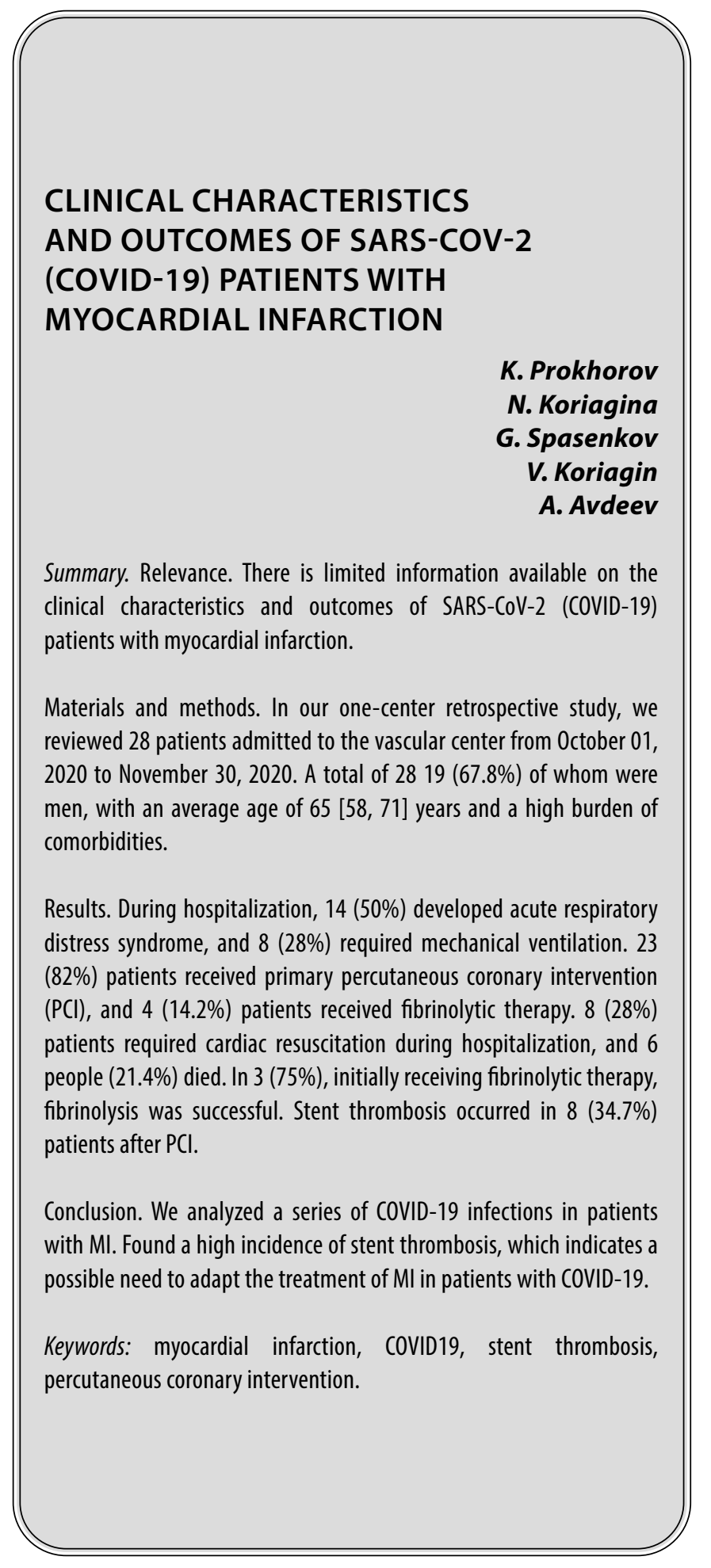

Прохоров Кирилл Владимирович

Соискатель, ФГБОУ ВО «Пермский государственный медицинский университет имени академика

Е.А. Вагнера» Минздрава России; главный врач ГБУЗ ПК ККД

kirpro059@gmail.com

Корягина Наталья Александровна

Д.м.н., профессор, ФГБОУ ВО «Пермский государственный медицинский университет имени академика Е.А. Вагнера» Минздрава России; главный терапевт Минздрава Пермского края nina11-85@mail.ru

Спасенков Григорий Николаевич Соискатель, ФГБОУ ВО «Пермский государственный медицинский университет имени академика

Е.А. Вагнера» Минздрава России, врач-кардиолог spy5502@mail.ru

Корягин Владимир Сергеевич

ФГБОУ ВО «Пермский государственный медицинский университет имени академика Е.А. Вагнера» Минздрава России vladimirkoryagin12@gmail.com Авдеев Алексей Викторович

К.м.н., дочент, ФГБОУ ВО «Пермский государственный медицинский университет имени академика Е.А. Вагнера» Минздрава России svenav@mail.ru

Аннотация. Актуальность. Имеется ограниченная информация о клинических характеристиках и исходах пациентов с заболеванием SARS-CoV-2 (COVID-19) с инфарктом миокарда.

Материалы и методы. В нашем одноцентровом ретроспективном исследовании мы рассмотрели 28 пациентов, госпитализированных в сосудистый центр с 01 октября 2020 года по 30 ноября 2020 года. Всего 2819 $(67,8 \%)$ из которых были мужчинами, со средним возрастом $65[58,71]$ лет и высоким бременем сопутствующих заболеваний.

Результаты. Во время госпитализации у 14 (50\%) развился острый респираторный дистресс-синдром, а 8 (28\%) потребовалась ИВЛ. 23 (82\%) пациентов получали первичное чрескожное коронарное вмешательство (ЧКВ), а 4 (14,2\%) фибринолитическую терапию. 8 (28\%) пациентов потребовалась реанимация сердца во время госпитализации, а 6 человек $(21,4 \%)$ умерли. У 3 (75\%), первоначально получавших фибринолитическую терапию, фибринолиз был успешным. Тромбоз стента произошел у 8 (34,7\%) пациентов после ЧКВ. 


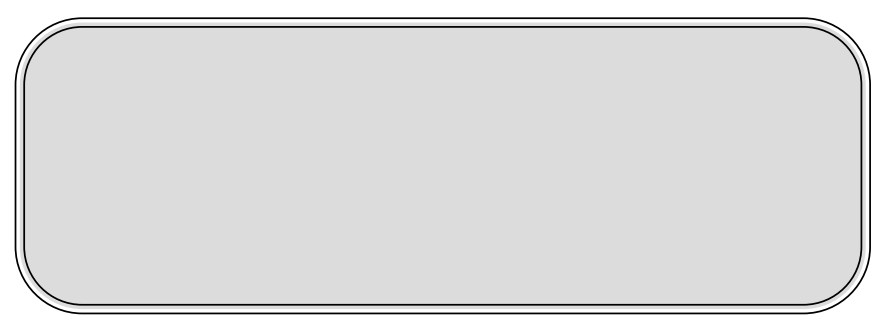

\section{Актуа^ьность}

П о состоянию на 20 мая 2020 года общее количество подтвержденных случаев заражения коронавирусом 2 (SARS-CoV-2 или COVID-19) тяжелого острого респираторного синдрома во всем мире превысило 4,5 миллиона случаев, а связанные с ними зарегистрированные смертельные случаи приблизились к 325000 [1]. Системные вирусные инфекции были связаны с острым инфарктом миокарда и воспалением $[2,3]$ и действительно может быть патофизиологическим триггером разрыва бляшки и тромбоза. Несмотря на растущее количество публикаций, касающихся общей популяции с положительным диагнозом COVID-19, мало что известно о конкретных клинических характеристиках и исходах пациентов с активной инфекцией COVID-19, у которых наблюдается инфаркт миокарда (ИМ). Фактически, данные до сих пор в основном ограничивались небольшой серией случаев из 18 пациентов в Нью-Йорке с зарегистрированной летальностью 72\% [4] и еще один из 28 пациентов из северной Италии с зарегистрированной летальностью $39 \%$ [5].

\section{Материал и методы}

Проведен ретроспективный одноцентровой обзор медицинских карт пациентов, последовательно госпитализированных в период с 01 октября 2020 г. по 30 ноября 2020 г. с COVID-19 и ИМ в региональный сосудистый центр (РСЦ) Пермского края. Пациенты были инфицированы COVID-19, который был подтвержден положительным результатом тестирования полимеразной цепной реакции из носоглотки. COVID-отрицательные пациенты, лечившиеся по поводу ИМ в РСЦ, были исключены из исследования. Сроки результатов ПЦР на COVID-19 в отношении времени чрескожного коронарного вмешательства (ЧКВ) не были доступны. Собранные данные включали демографические данные пациентов, сопутствующие заболевания, существующие лекарства, лабораторные тесты, результаты электрокардиограммы, диагноз во время госпитализации, лечение в стационаре, лечение (фибринолитическая терапия, ЧКВ, использование вазопрессоров) и исходы (продолжительность госпитализации, успешность реваскуляризации). Сообщалось о приеме лекарств в домашних условиях на основании согласования при-
Заключение. Мы провели анализ серии случаев инфицирования COVID-19 пациентов с ИМ. Обнаружили высокую частоту тромбоза стента, что указывает на возможную необходимость адаптации лечения ИМ у пациентов c COVID-19.

Ключевые слова: инфаркт миокарда, COVID19, тромбоз стента, чрезкожное коронарное вмешательство.

ема лекарств, проведенного врачом в приемном отделении. Диагноз ишемической болезни сердца включал перенесенный ранее инфаркт миокарда (ПИКС), операцию аортокоронарное шунтирование, предыдущие чрезкожные коронарные вмешательства (ЧКВ) и наличие стенокардии.

Выбор первоначальной стратегии реперфузии фибринолитическая терапия или первичное ЧКВ оставался на усмотрение лечащего врача в отделении в соответствии с клиническими рекомендациями, поскольку для таких пациентов не существует иных международных рекомендаций по ведению больных с COVID-19. Как правило, пациенты с COVID-19 и ИМ в РСЦ получали инвазивую терапию. Успешный фибринолиз определялся как смещение сегмента ST более чем на 50\% в течение 60-90 мин после фибринолитического введения при отсутствии боли в груди и признаков гемодинамической или электрической нестабильности. Эффективность реваскуляризации при инфаркте миокарда (TIMI) оценивался ангиографически лечащим врачом [6]. ИМ и тромбоз стента определялись согласно четвертому универсальному определению ИМ [7].

Инфекция COVID-19 была классифицирована в соответствии с определениями ВОЗ клинических синдромов, связанных с COVID-19 [8]. Определение стадии хронической болезни почек проводилось в соответствии с рекомендациями по клинической практике [9].

Непрерывные переменные представлены в виде медианы [25- й процентиль, 75- й процентиль]. Категориальные переменные представлены как частота и процент.

\section{Полученные результаты}

В этот обзор было включено 28 пациентов, большинство из которых составляли мужчины, средний возраст которых составлял $65[58,71]$ лет.

Пациенты несли высокую коморбидную нагрузку; у большинства $(15,55 \%)$ было не менее 3 сопутствующих заболеваний. Наиболее частыми сопутствующими заболеваниями были: сахарный диабет (16, 57,1\%), 
Таблица 1. Распространенность сопутствующей патологии у пациентов с инфарктом миокарда c COVID-19

\begin{tabular}{|l|l|l|}
\hline Сопутствующие заболевания & Абс., чел. & $\%$ \\
\hline Сахарный диабет & 16 & 57,1 \\
\hline Артериальная гипертензия & 24 & 85,7 \\
\hline Хроническая болезнь почек & 12 & 42,9 \\
\hline $\begin{array}{l}\text { Хроническая обструктивная болезнь } \\
\text { легких }\end{array}$ & 8 & 28,6 \\
\hline Бронхиальная астма & 3 & 10,7 \\
\hline Злокачественное новообразование & 2 & 7,1 \\
\hline Фибрилляция предсердий & 12 & 42,9 \\
\hline
\end{tabular}

Таблица 2. Локализация инфаркта миокарда с COVID-19

\begin{tabular}{|l|l|l|}
\hline Локализация & Абс., чел. & $\%$ \\
\hline Передне-латеральный & 12 & 44,4 \\
\hline Задне-латеральный & 12 & 44,4 \\
\hline Задне-базальный & 1 & 3,7 \\
\hline Передне-перегородочный & 1 & 3,7 \\
\hline Передне-боковой & 2 & 7,4 \\
\hline Задне-боковой & 0 & 0 \\
\hline
\end{tabular}

Таблица 3. Осложенения инфаркта миокарда с COVID-19

\begin{tabular}{|l|l|l|}
\hline Показатель & Абс., чел. & $\%$ \\
\hline Наличие отека легких & 13 & 46,4 \\
\hline Наличие кардиогенного шока & 6 & 21,4 \\
\hline Потребность в вазопрессорах & 6 & 21,4 \\
\hline Потребность в внутревенных дуиретиках & 15 & 53,6 \\
\hline
\end{tabular}

артериальная гипертензия (24, 85,7\%), фибрилляция предсердий $(12,42,9 \%)$.

Во время госпитализации у 12 пациентов (42,8\%) была легкая пневмония, у 14 (50\%) развился острый респираторный дистресс-синдром, а 8 (28\%) потребовалась инвазивная вентиляция легких (ИВЛ).

Среднее время реперфузии от боли составило 12,6 $[8,15]$ часов. При этом 23 (82\%) пациентов получали первичное чрескожное коронарное вмешательство (ЧКВ), при этом тромбоз стента произошел у 8 (34,7\%) пациентов после ЧКВ.

Из 22 пациентов, получавших первичное ЧКВ, у 1 пациента (4,3\%) не было признаков обструкции коронарной артерии, и у него был диагностирован миокардит. Среднее время от двери до провода составляло 128 [90, 210] минут.
Наиболее частой локализацией была передне-латеральная $(44,4 \%)$ и задне-латеральная $(44, \%)$.

При ЧКВ всем пациентам был установлен стент с лекарственным покрытием. 7 пациентам (30,4\%) потребовалась инвазивная искусственная вентиляция легких. Кроме того, у 6 (26\%) пациентов потребовалась реанимация сердца, а $5(21,7 \%)$ умерли.

Из 4 пациентов, первоначально получавших фибринолитическую терапию, 3 пациентов (85\%) имели успешный фибринолиз. Среднее время реперфузии составило $27[20,34]$ минут. У всех пациентов использовалась альтеплаза.

Из всех 28 пациентов с ИМпST, положительных на инфекцию COVID-19, 8 (28\%) потребовалась инвазивная искусственная вентиляция легких. Их средний возраст составлял 68 [60, 74] лет, и 6 (75\%) были мужчинами. 
Наиболее частыми сопутствующими заболеваниями были ишемическая болезнь сердца в анамнезе $(6,75 \%)$, артериальная гипертензия $(8,100 \%)$ и ХОБЛ $(4,50 \%)$.

Семь (87\%) пациентов, которым потребовалась искусственная вентиляция легких, перенесли первичное ЧKB, а 1 (12,5\%) пациент получал фибринолитическую терапию в качестве начальной стратегии реперфузии.

При этом у 7 (75\%) потребовалась реанимация сердца, 6 (75\%) умерли. Средняя продолжительность пребывания составила $14[9,15]$ дней.

\section{Обсужление}

Оптимальная стратегия ведения пациентов с ИМ и сопутствующим COVID-19 может представлять большую проблему, чем для пациентов с ИМ вне пандемии.

По данным литературы, имеются рекомендации использования фибринолитической терапии вместо первичного чрескожного коронарного вмешательства у пациентов, которые обращаются в течение 12 часов с момента появления симптомов и не имеют противопоказаний для фибринолитиков [10].

Наше исследование заполняет критический пробел в знаниях, описывая исходные характеристики и сопутствующие заболевания,, клиническое течение, стратегии реваскуляризации и исходы пациентов, госпитализированных с COVID-19 и ИМ.

Пациенты в исследовании были пожилого возраста и имели высокую сопутствующую патологию. Все пациенты имели по крайней мере 1 сопутствующую патологию, почти все имели 2 или более сопутствующих заболеваний, а многие имели 3 и более сопутствующих заболевания.

Летальность в этом исследовании составила 21,4\%. Но мы зафиксировали высокий уровень смертности среди пациентов с инвазивной вентиляцией легких в пределах ранее обсужденных показателей среди пациентов с COVID-19 в критическом состоянии в Китае и США [11].
Инфаркт миокарда - тяжелая сосудистая катастрофа, и на смертность при этом заболевании влияет множество характеристик пациента. Например, женский пол, пожилой возраст, передний ИМ и перенесенный инфаркт миокарда связаны с худшими исходами у этих пациентов [12]. Сообщается, что грипп и другие респираторные вирусные инфекции действуют как триггеры ИМ [2] и грипп может незначительно увеличить риск сосудистой катастрофы [3]. Следовательно, вполне вероятно, что инфекция COVID-19 и системная воспалительная реакция во многих случаях могли быть спусковым механизмом для коронарного события.

Стандарт лечения пациентов без COVID-19 с ИM это инвазивная реваскуляризация в течение 90 минут после обращения в больницу. Фибринолитическая терапия рекомендуется, если ожидается задержка до инвазивной реваскуляризации более чем на 90 минут [7].

Вызывает тревогу то, что пациенты в этом исследовании, которые лечились с помощью ЧКВ, имели частоту тромбоза стента 34,7\%, что намного выше, чем ранее сообщавшаяся частота раннего тромбоза стента, равная 1\% [13]. Кроме того, сообщалось, что пациенты в отделении интенсивной терапии по поводу COVID-19 имеют чрезвычайно высокую частоту тромботических осложнений (примерно 31\%) [14] и что более интенсивная антикоагуляция может улучшить результаты у таких пациентов с повышенным уровнем D-димера. Эти специфические для COVID-19 наблюдения могут объяснить, почему пациенты в нашем исследовании имели более высокий, чем ожидалось, уровень тромбоза стента. Следовательно, важно продолжить мониторинг тромботических событий в этой популяции с помощью более крупных исследований, чтобы определить время и стратегии инвазивной реваскуляризации.

Это исследование имеет все ограничения ретроспективных исследований проспективно собранных данных.

В заключение, мы обнаружили высокий уровень тромботических осложнений и очень высокую смертность. Наши результаты предполагают возможную необходимость адаптации лечения ИМ для пациентов с сопутствующей инфекцией COVID-19.

\section{ЛИТЕРАТУРА}

1. The Center for Systems Science and Engineering (CSSE) at Johns Hopkins University Coronavirus COVID-19 global cases. 2020 https://gisanddata.maps.arcgis. com/apps/opsdashboard/index.html\#/bda7594740fd40299423467b48e9ecf6. Accessed May 20, 2020).

2. Warren-Gash C, Hayward AC, Hemingway H, Denaxas S, Thomas SL, Timmis AD, Whitaker H, Smeeth L. Influenza infection and risk of acute myocardial infarction in England and Wales: a CALIBER self-controlled case series study. J Infect Dis. 2012; 206: 1652-1659.

3. Claeys MJ, Coenen S, Colpaert C, Bilcke J, Beutels P, Wouters K, Legrand V, Van Damme P, Vrints C. Environmental triggers of acute myocardial infarction: results of a nationwide multiple-factorial population study. Acta Cardiol. 2015; 70: 693-701. 
4. Bangalore S., Sharma A., Slotwiner A., Yatskar L., Harari R., Shah B., Ibrahim H., Friedman G.H. ST-Segment elevation in patients with Covid-19 — a case series [published online ahead of print, 2020 Apr 17]. N Engl J Med. 2020; (NEJMc2009020).

5. Zeng J, Huang J, Pan L. How to balance acute myocardial infarction and COVID-19: the protocols from Sichuan Provincial People's Hospital [published online March 11, 2020].Intensive Care Med. doi: 10.1007/s00134-020-05993-9.

6. Chesebro JH., Knatterud G, Roberts R, Borer J, Cohen LS, Dalen J, Dodge HT, Francis CK, Hillis D, Ludbrook P. Thrombolysis in Myocardial Infarction (TIMI) Trial, Phase I: A comparison between intravenous tissue plasminogen activator and intravenous streptokinase. Clinical findings through hospital discharge. Circulation. 1987; 76: 142-154.

7. Thygesen K, Alpert JS, Jaffe AS, Chaitman BR, Bax JJ, Morrow DA, White HD. Executive Group on behalf of the Joint European Society of Cardiology (ESC). American College of Cardiology (ACC) American Heart Association (AHA) World Heart Federation (WHF) Task Force for the Universal Definition of Myocardial Infarction Fourth Universal Definition of Myocardial Infarction (2018). J Am Coll Cardiol. 2018; 72: 2231-2264.

8. Clinical management of severe acute respiratory infection (SARI) when COVID-19 disease is suspected. World Health Organization, Published March 13, 2020 https://www.who.int/docs/default-source/coronaviruse/clinical-management-of-novel-cov.pdf.

9. National Kidney Foundation K/DOQI clinical practice guidelines for chronic kidney disease: evaluation, classification, and stratification. Am J Kidney Dis. 2002; 39: S1-S266.

10. Zeng J, Huang J, Pan L. How to balance acute myocardial infarction and COVID-19: the protocols from Sichuan Provincial People's Hospital [published online ahead of print, 2020 Mar 11]. Intensive Care Med. 2020; 46: 1111-1113.

11. Huang C, Wang Y, Li X, Ren L, Zhao J, Hu Y. Clinical features of patients infected with 2019 novel coronavirus in Wuhan, China [published correction appears in Lancet. 2020 Jan 30]. Lancet. 2020; 395: 497-506.

12. Vasiljević Z, Stojanović B, Kocev N. [Hospital mortality trend analysis of patients with ST elevation myocardial infarction in the Belgrade area coronary care units]. Srp Arh Celok Lek. 2008; 136: 84-96.

13. Levine GN, Bates ER, Blankenship JC, Bailey SR2011 ACCF/AHA/SCAI Guideline for Percutaneous Coronary Intervention: executive summary: a report of the American College of Cardiology Foundation/American Heart Association Task Force on Practice Guidelines and the Society for Cardiovascular Angiography and Interventions. Catheter Cardiovasc Interv. 2012; 79: 453-495.

14. Klok FA, Kruip MJHA, van der Meer NJM, Arbous MS Incidence of thrombotic complications in critically ill ICU patients with COVID-19 [published online ahead of print, 2020 Apr 10]. Thromb Res. 2020; (S0049-3848(20)30120-1)

( П Прохоров Кирилл Владимирович ( kirpro059@gmail.com ), Корягина Наталья Александровна ( nina1 1-85@mail.ru ),

Спасенков Григорий Николаевич ( spy5502@mail.ru ), Корягин Владимир Сергеевич ( vladimirkoryagin12@gmail.com ),

Авдеев Алексей Викторович ( svenav@mail.ru ).

Журнал «Современная наука: актуальные проблемы теории и практики» 